\title{
An Investigation of Important Gas-Phase Reactions of Nitrogenous Species from the Simulation of Experimental Measurements in Combustion Systems
}

\author{
K. J. HUGHES, A. S. TOMLIN*, E. HAMPARTSOUMIAN, and W. NIMMO \\ Department of Fuel and Energy, University of Leeds, Leeds, LS2 9JT, UK
}

I. G. ZSÉLY, M. UJVÁRI, and T. TURÁNYI

Department of Physical Chemistry, Eötvös University (ELTE), P.O. Box 32, H-1518 Budapest, Hungary and Chemical Research Center, P.O. Box 17, H-1525 Budapest, Hungary

A. R. CLAGUE**, and M. J. PILLING

School of Chemistry, University of Leeds, Leeds, LS2 9JT, UK

\begin{abstract}
Simulated results from a detailed elementary reaction mechanism for nitrogen-containing species in flames consisting of hydrogen, $\mathrm{C}_{1}$ or $\mathrm{C}_{2}$ fuels are presented, and compared with bulk experimental measurements of nitrogen-containing species in a variety of combustion systems including flow reactors, perfectly stirred reactors, and low pressure laminar flames. Sensitivity analysis has been employed to highlight the important reactions of nitrogenous species in each system. The rate coefficients for these reactions have been compared against the expressions used in three other recent reaction mechanisms: version 3.0 of the GRI mechanism, the mechanism of Glarborg, Miller and co-workers, and that of Dean and Bozzelli. Such comparisons indicate that there are still large discrepancies in the reaction mechanisms used to describe nitrogen chemistry in combustion systems. Reactions for which further measurements and evaluations are required are identified and the differences between the major mechanisms available are clearly demonstrated.
\end{abstract}

\section{INTRODUCTION}

Modelling is an important tool in developing an understanding of the processes generating and removing NOx in combustion systems. The chemical mechanism for the gas phase production and destruction of NOx is an important part of the design of low NOx systems. Several groups [1-3] have attempted to develop comprehensive schemes or components of schemes describing NOx formation and to validate these against experimental measurements. It is sometimes assumed that NOx chemistry is well understood; this might lead to the conclusion that the available comprehensive mechanisms are almost equivalent. It is certainly true that the major pathways for NOx formation have been identified and that a large number of the elementary reactions involved in NOx chemistry have been extensively studied. Some of the rate coefficients have been evaluated by the CEC (Commission of European Communities) group

\footnotetext{
*Corresponding author. E-mail: alisont@chem.leeds.ac.uk **Currently at DERA, DERA Pyestock, Farnborough, Hants. GU14 OLS, UK
}

evaluating kinetic data for combustion modeling, providing temperature and pressure-dependent rate coefficients with uncertainty estimates [4] and henceforth referred to as the CEC evaluation. However, as this paper demonstrates, there are large differences between some of the rate coefficients used in the most comprehensive mechanisms. There are also experimental measurements for which the predictions of even the optimized mechanisms do not perform well. The comparisons indicate that gas phase NOx chemistry is not as well understood as is often assumed, and that developments in NOx mechanisms will require more than just fine tuning. One aim of this work is to provide information for identifying areas where development is necessary.

The process of developing and evaluating a mechanism is continuous and time-consuming. Rate coefficients are often re-measured, recalculated, or re-evaluated and therefore a comprehensive mechanism must be updated to reflect these improvements. The time involved means that it is not feasible to re-evaluate a mechanism on the basis of changes in individual parameters, so a mechanism is generally up- 
dated when a substantial number of changes have to be made to kinetic or thermodynamic parameters. A good example is the GRI reaction set, where the mechanism is periodically updated and re-evaluated, when parameters are re-estimated and new measurements become available.

The core of the GRI mechanism, originally developed by Frenklach et al. [5-8], is for the oxidation of methane. This has been tested against a large set of experimental observations and many of the rate coefficients in the mechanism have been tuned to improve the agreement between the simulated results and the experimental measurements. Version 2.11 of this mechanism includes nitrogen chemistry relevant to the burning and reburning of natural gas, although the nitrogen chemistry was at a lower level of optimization than the methane mechanism. The current version, GRI-mech 3.0 [2] improves upon version 2.11 in several respects. In the case of the nitrogen chemistry, additional experiments on prompt NO formation and reburn in a shock tube, low pressure flame, and a flow reactor have been added.

Glarborg et al. [1] have constructed a detailed chemical mechanism for the oxidation of $\mathrm{C} 1$ and C2 hydrocarbons, hydrogen cyanide, ammonia, and the interactions between carbon and nitrogenous species. The purpose of the mechanism was to investigate reburn chemistry under flow reactor conditions. Recently, nitrogen chemistry has been reviewed [3] to provide the best rate coefficients, based on an evaluation of the measured rate coefficients and also the product channels of individual elementary reactions, with theoretical calculations of rate coefficients where necessary.

Hughes et al. [9, 10] have created a comprehensive chemical mechanism for the oxidation of methane. This mechanism has been extended by adding a section describing $\mathrm{NO}_{\mathrm{x}}$ chemistry. The combined mechanism is henceforth referred to as the Leeds $\mathrm{NO}_{\mathrm{x}}$ mechanism, and is available via the World Wide Web [10, 11]. The aim in creating this mechanism was complementary to that of GRI, which tunes rate coefficients within uncertainty limits to generate an optimized mechanism for combustion problems. The Leeds mechanism aims to use, wherever possible, evaluated rate coefficients with mini- mal tuning. Coupled with testing against experimental measurements, sensitivity analysis and an examination of differences from other mechanisms, such a mechanism enables one to assess the reliability of currently available rate coefficients for elementary reactions. The use of evaluated rate coefficients, where available, has the advantage that it is based on the best assessment of rate coefficients and channel efficiencies. Its weakness is that substantial, comprehensive evaluations are infrequent, so that the most recent measurements may be excluded. In addition, evaluations are rarely fully comprehensive, so that some ad hoc assessment of rate parameters is necessary in constructing a mechanism.

The next section evaluates the mechanism by comparing its predictions against a large experimental data set covering flame profiles, batch and jet-stirred reactors. The measurements were chosen from the GRI suite. The section on 'Sensitivity Analysis' includes a comprehensive study of the sensitivities of nitrogenous species over a wide range of experimental conditions and lists those reactions influencing [NOx] profiles in a general sense. In 'Discussion', the rate coefficients of these reactions are compared for the four reaction mechanisms discussed above. This comparison highlights the major differences between the mechanisms and so provides useful information for tuning predictions using the most poorly characterized reactions with a high sensitivity under certain conditions. 'Simulation of Bulk Experimental Measurements' and 'Sensitivity Analysis' demonstrate where further measurements and evaluations are required, and reveal the differences between the major mechanisms available.

\section{SIMULATION OF BULK EXPERIMENTAL MEASUREMENTS}

In their optimization procedure, the GRI group [2] use a large collection of measurements for nitrogenous species in combustion systems termed the "target data" set. The data set is chosen to provide a wide range of experimental conditions from available measurements. A selection of these from laminar flames [12-16], flow tubes [17], and jet-stirred reactors [18, 19] 
were simulated to provide a wide variety of conditions to fully test the chemical mechanism. The CHEMKIN program [20] has been used to simulate the bulk experimental data. Flow reactors, perfectly stirred reactors, and laminar flames have been simulated using the SENKIN [21], PSR [22], and PREMIX [23] modules, respectively. Sensitivity information from the CHEMKIN modules has been processed using KINALC [24], a Fortran program, which provides ordered lists of sensitivities for selected species.

\section{Laminar Flame Species Profiles}

A selection of experimentally measured concentrations in low pressure laminar flames has been simulated. Different fuels were used, and concentration profiles for $\mathrm{NO}, \mathrm{CN}, \mathrm{HCN}, \mathrm{N}_{2}, \mathrm{~N}_{2} \mathrm{O}$, and NCO have been measured [12-16]. Figures 1-5 show both the experimental and predicted results. In all cases, except where stated otherwise, symbols represent experimental data, solid lines represent our simulation using the experimental temperature, pressure and mass flow rates, and dashed lines show, for comparison, the simulations from GRI-mech 3.0 [2]. Figures 1 and 2 demonstrate predictions of lean $(\Phi=$ $0.5)$ and rich $(\Phi=1.5)$ flames [12] of hydrogen+oxygen+argon at 25 Torr doped with $\mathrm{HCN}$, and a rich $(\Phi=1.13)$ methane/air flame [13] at 30 Torr. There is satisfactory agreement for both the lean and rich flames. Figure 3 shows the predictions of relative mole fractions for the intermediates $\mathrm{CN}, \mathrm{NH}$ and $\mathrm{NCO}$ in a stoichiometric flame of methane+oxygen + argon [14] at 10 Torr doped with either $\mathrm{NO}$ or $\mathrm{N}_{2} \mathrm{O}$. Again there is good agreement for the relative changes in concentration caused by the dopant. The predictions of $[\mathrm{NH}]$ and [NO] in Fig. 4 for a stoichiometric hydrogen/nitrous oxide/argon flame at 20 Torr [15], although not perfect, are in reasonable agreement. Figure 5 represents an experiment that was designed to highlight a specific route forming NOx, via the NNH intermediate in a rich $(\Phi=1.5)$ hydrogen/air flame at 38 and 78 Torr [16]. The over-prediction of [NO] in this case is the worst of all the predictions by both the Leeds and GRI-mech 3.0. We shall return to these predictions in the discussion.
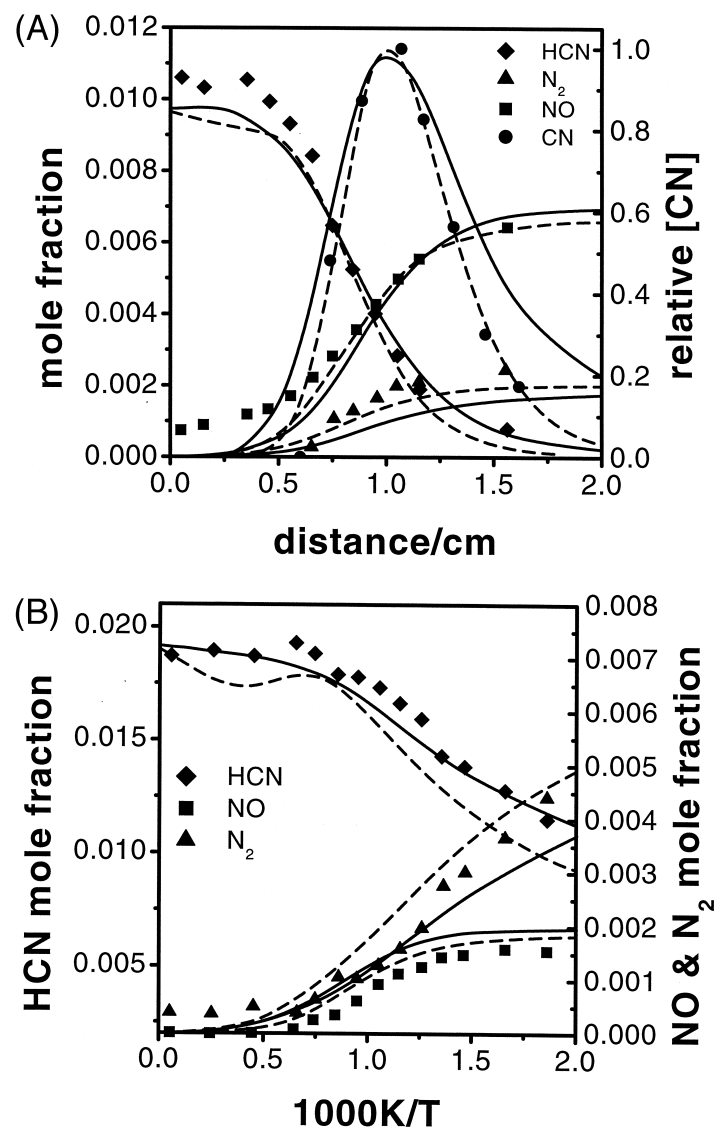

(C)

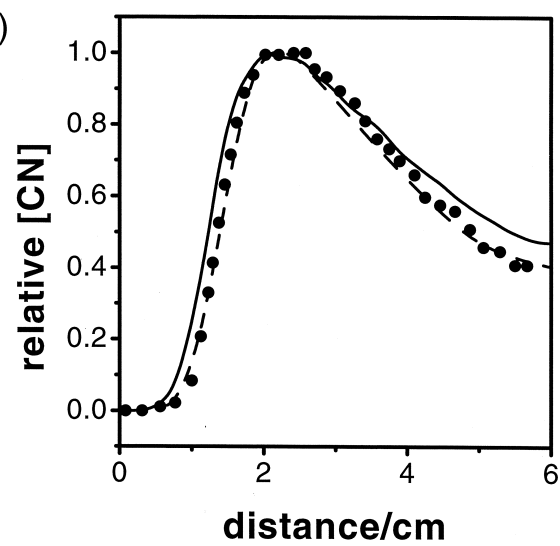

Fig. 1. Species profiles in an $\mathrm{H}_{2} / \mathrm{O}_{2} / \mathrm{HCN} / \mathrm{Ar}$ flame at 25 Torr [12]: (a) $\mathrm{HCN}, \mathrm{CN}, \mathrm{NO}$ and $\mathrm{N}_{2}$ in a lean $(\Phi=0.5)$ flame; (b) $\mathrm{HCN}, \mathrm{NO}$ and $\mathrm{N}_{2}$ in a rich $(\Phi=1.5)$ flame; (c) $\mathrm{CN}$ profile in a rich $(\Phi=1.5)$ flame.

\section{Flow Tube Data}

Glarborg and Miller [17] have studied the oxidation of $\mathrm{HCN}$ in a flow reactor at various temperatures, residence times and compositions 


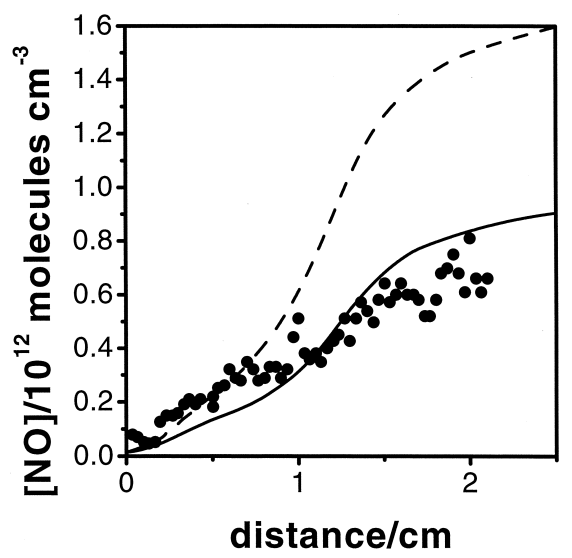

Fig. 2. $[\mathrm{NO}]$ in a rich $(\Phi=1.15)$ methane/air flame at 30 Torr [13].

at a total pressure of 1.05 atm. Figures $6 \mathrm{a}$ and $6 \mathrm{~b}$ show the experimental and simulated concentrations for the nitrogen-containing species. In general, the agreement is good for those experiments that include $\mathrm{CO}$, and less so for those based only on HCN or HCN and NO.

\section{Jet-Stirred Reactor Data}

Bartok et al. [18] have measured mole fractions of NO in the outlet of a jet-stirred reactor oxidising methane either undoped or doped with $\mathrm{NO}$, at $1 \mathrm{~atm}$, and $\Phi$ from 0.65 to 1.5 . Figures $7 \mathrm{a}$ and $7 \mathrm{~b}$ show both the experimental and predicted values plotted against $\Phi$. The simulations show good agreement for lean conditions in Fig. 7a, and the destruction of NO for the doped flames in Fig. 7b. The agreement is less good in Figs. 7a and $7 \mathrm{~b}$ under rich conditions, where a large percentage of NO is being destroyed when compared to the experimental profiles for the doped flames. Steele et al. [19] have also investigated the formation of $\mathrm{NO}$ and $\mathrm{N}_{2} \mathrm{O}$ in a jet-stirred reactor from a lean $(\Phi=$ $0.52-0.62$ ) mixture of methane and air at $1 \mathrm{~atm}$. The agreement between computed and measured values in Fig. 8 is good, with only a slight over-prediction of [NO] across the temperature range.

\section{SENSITIVITY ANALYSIS}

The KINALC program [24] was used to process the sensitivity coefficients generated from the
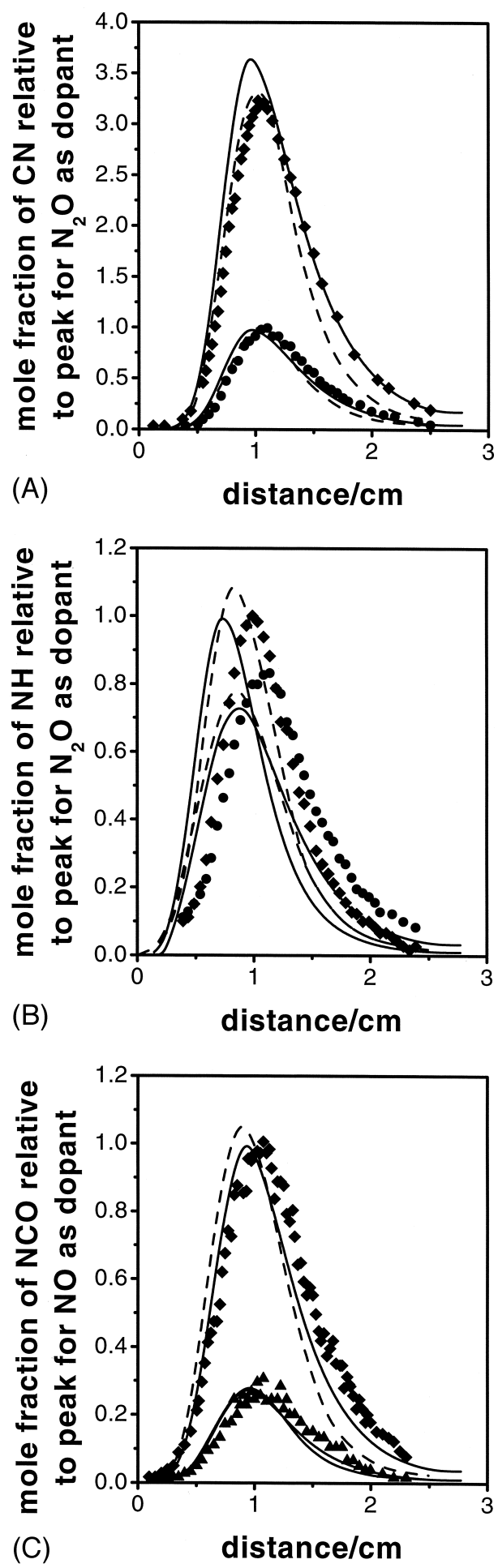

Fig. 3. Concentration profiles in a 10 Torr stoichiometric $\mathrm{CH}_{4} / \mathrm{O}_{2} /$ Ar flame [14] doped with $\mathrm{NO}$ or $\mathrm{N}_{2} \mathrm{O}$ for: (a) $\mathrm{CN}$; (b) $\mathrm{NH}$; (c) NCO. 


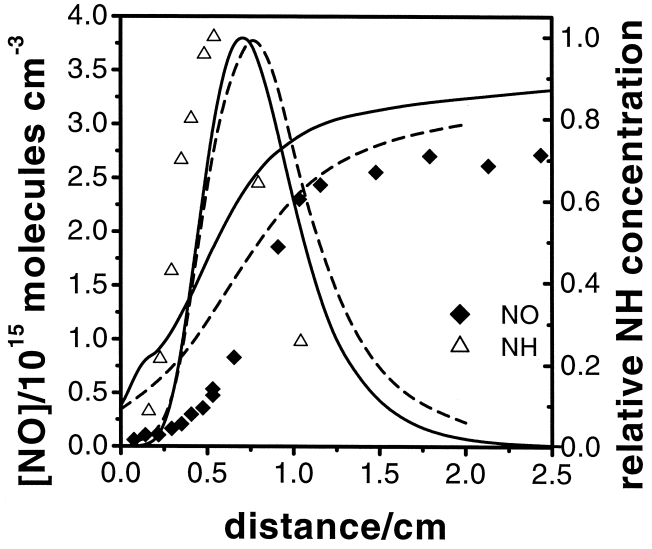

Fig. 4. $[\mathrm{NO}]$ and $[\mathrm{NH}]$ profiles in a 20 Torr stoichiometric $\mathrm{H}_{2} / \mathrm{N}_{2} \mathrm{O} /$ Ar flame [15].

CHEMKIN modules. In each case, for all the experimentally observed nitrogenous species, a ranked list of concentration sensitivities was generated. In the simulations of laminar flames, sensitivities were compiled over the appropriate range of height above the burner. For the jet-stirred reactor and flow tube simulations, the ranked lists of sensitivities were compiled at a variety of conditions of stoichiometry and temperature, chosen to cover all of the variations in predicted behavior. From each of these lists, the ten reactions with the highest absolute sensitivities, were selected, and reactions involving nitrogen identified. It should be pointed out that for certain conditions some forward/reverse reaction pairs can be identified that have high sensitivities of opposite sign and almost equal

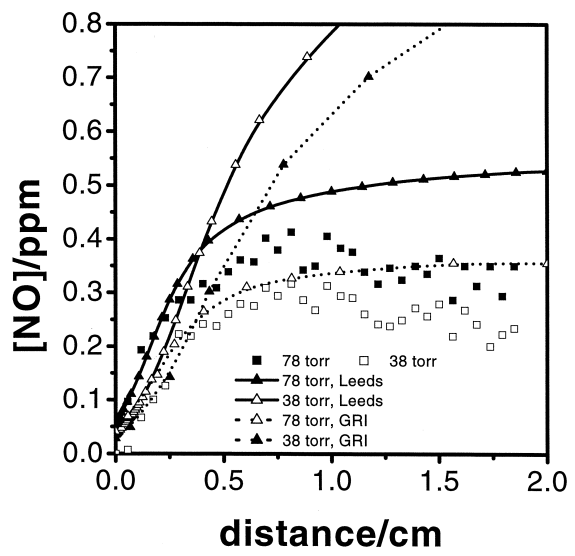

Fig. 5. [NO] profiles in a rich $(\Phi=1.5) \mathrm{H}_{2}$ /air flame at 38 and 78 Torr [16].
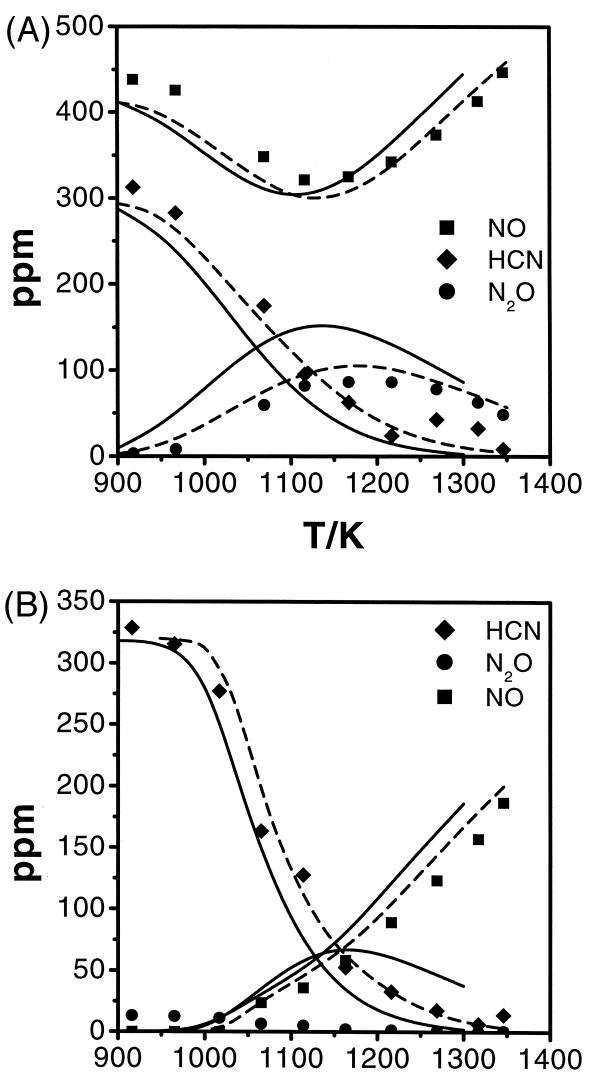

$\mathrm{T} / \mathrm{K}$

Fig. 6. $[\mathrm{HCN}],[\mathrm{NO}]$ and $\left[\mathrm{N}_{2} \mathrm{O}\right]$ from $\mathrm{HCN}$ oxidation in a flow reactor at $1.05 \mathrm{~atm}$ : (a) $298 \mathrm{ppm} \mathrm{HCN}, 1620 \mathrm{ppm} \mathrm{CO}$, $434 \mathrm{ppm} \mathrm{NO}, 2.3 \% \mathrm{O}_{2}, 2.6 \% \mathrm{H}_{2} \mathrm{O}$, balance $\mathrm{N}_{2}$; (b) $303 \mathrm{ppm}$ $\mathrm{HCN}, 447$ ppm NO, 2.3\% $\mathrm{O}_{2}, 2.7 \% \mathrm{H}_{2} \mathrm{O}$, balance $\mathrm{N}_{2}$.

magnitude. The high ranking of these reactions is artificial, as any change caused by the modification in such rate parameters will be mirrored by a compensating change in the reverse reaction, given a fixed equilibrium constant. These reversible reactions have been eliminated in pairs from the ordered tables, so that they do not influence the ranking of other reactions. Table 1 lists the reactions involving nitrogenous species found to be important for each of the experimentally observed nitrogenous species. This information helps to indicate which groups of reactions are important for different conditions in each data set. We have omitted detailed sensitivity plots for the whole range of conditions because the amount of data obscures useful information. Table 1 also gives an indication of the level of agreement between the rate coefficient expressions between 500 to 2000 

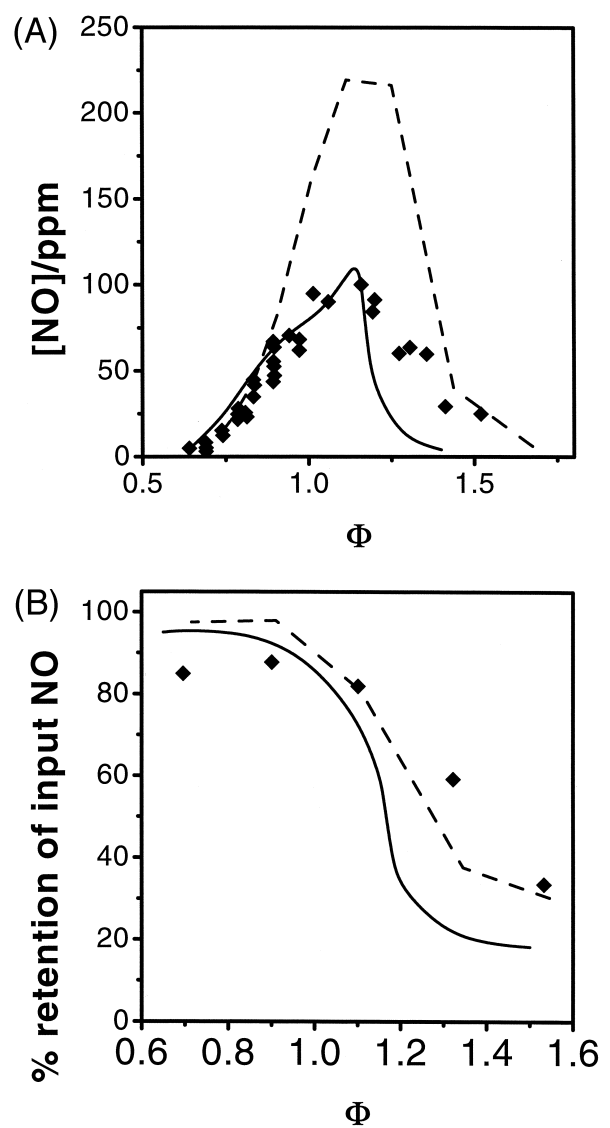

Fig. 7. [NO] in a jet-stirred reactor fed with methane and air mixture at 1 atm at 1655 to $2020 \mathrm{~K}$ [18]: (a) NO production; (b) NO retention when doped with $1300 \mathrm{ppm}$ of NO.

$\mathrm{K}$ for the different reaction mechanisms under discussion. In certain cases, where the rate coefficients are too small at low temperatures to be meaningful, the temperature range of interest is restricted to between 1000 to $2000 \mathrm{~K}$. A cross in the table indicates where the rate parameter we have used differs by more than a factor of three from the other mechanisms. A hash indicates rate parameters within a factor of three, a plus indicates identical or negligibly different rate parameters, whereas a dash indicates that the particular reaction or product channel is absent from the mechanism. This table provides a clear overview of the important reactions for each set of conditions and facilitates comparison of the four mechanisms. Table 2 lists the rate coefficient expressions used in the Leeds NOx mechanism for each reaction included in Table 1. The full mechanism is available on the World Wide Web [10, 11].

\section{DISCUSSION}

In general, the agreement between the Leeds NOx mechanism and the experimental measurements is satisfactory, although there are some differences for particular conditions. Some discrepancies may be due to experimental error or to a lack of information on detailed experimental conditions, e.g., heat loss or radical loss at the burner. Other differences are clearly due to the mechanism and we will discuss these here. Each of the four mechanisms gives good agreement with experiment for certain data sets and less good for others, perhaps because of the way they have been constructed or tuned. The areas of agreement are different for each mechanism, however, and the data given in Table 2 helps to highlight differences between them. We now discuss these differences using reaction groups involving individual species. In Figs. 9-15 the labeling is as follows; L, GRI, GM, and DB denote the mechanisms of Leeds [11], GRI-mech 3.0 [2], Glarborg and Miller [1], and Dean and Bozzelli [3]. Numbers in square brackets refer to the reference for the source of the rate coefficient parameters.

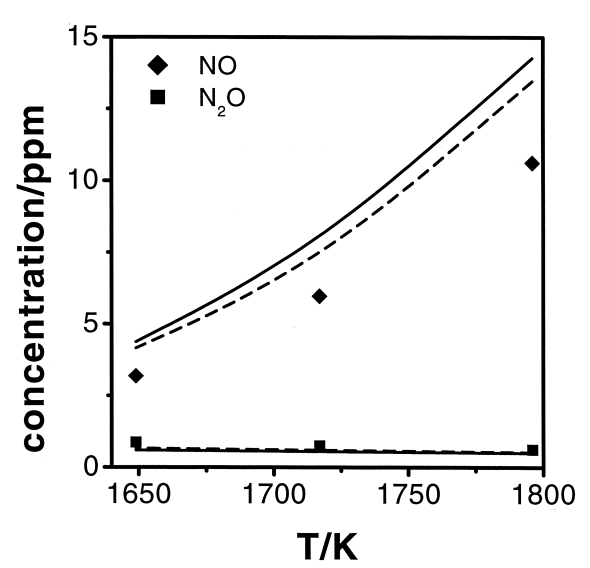

Fig. 8. $[\mathrm{NO}]$ and $\left[\mathrm{N}_{2} \mathrm{O}\right]$ in a jet-stirred reactor fed with a lean $(\Phi=0.52-0.62)$ mixture of methane and air at $1 \mathrm{~atm}$ [19]. 


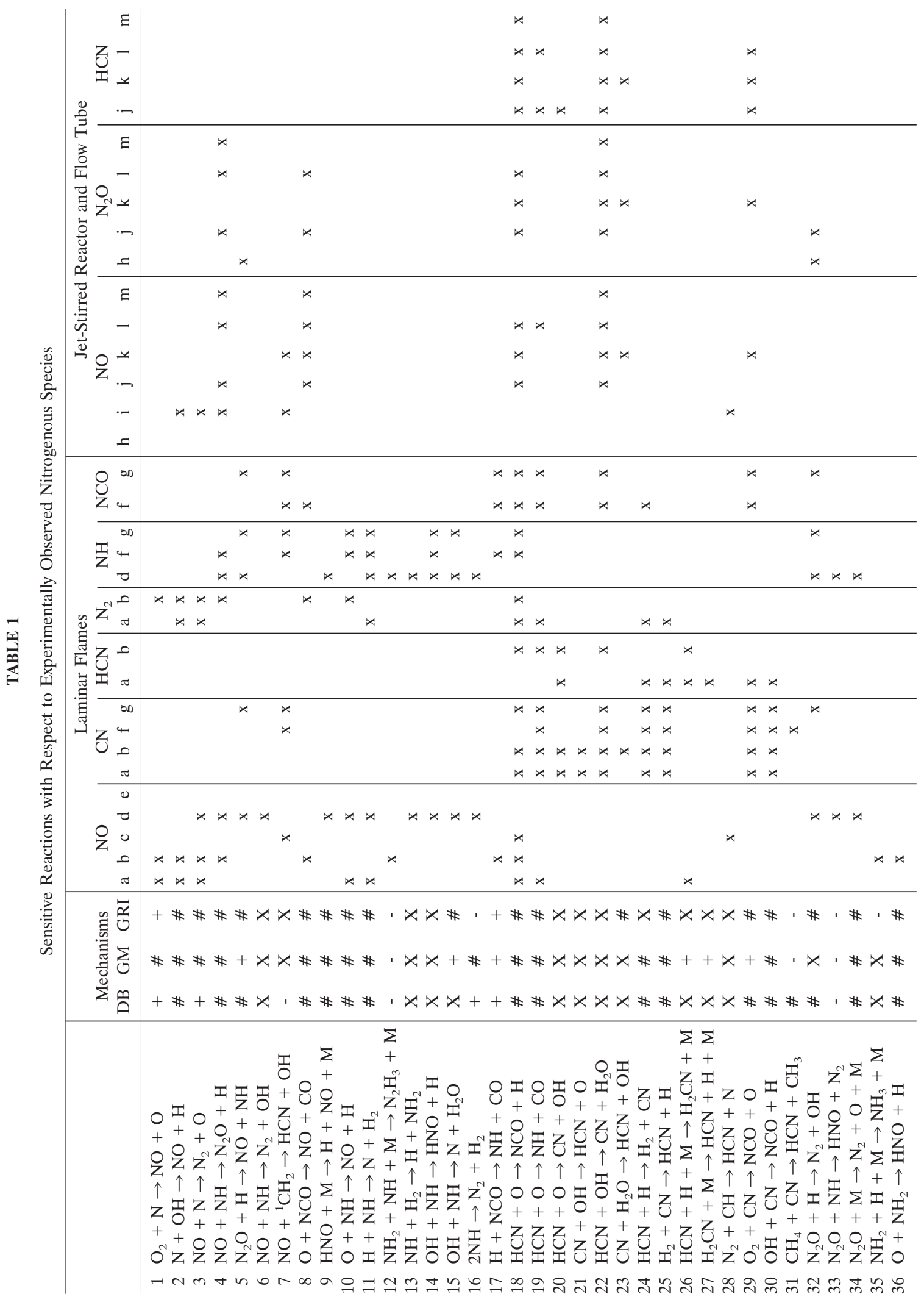




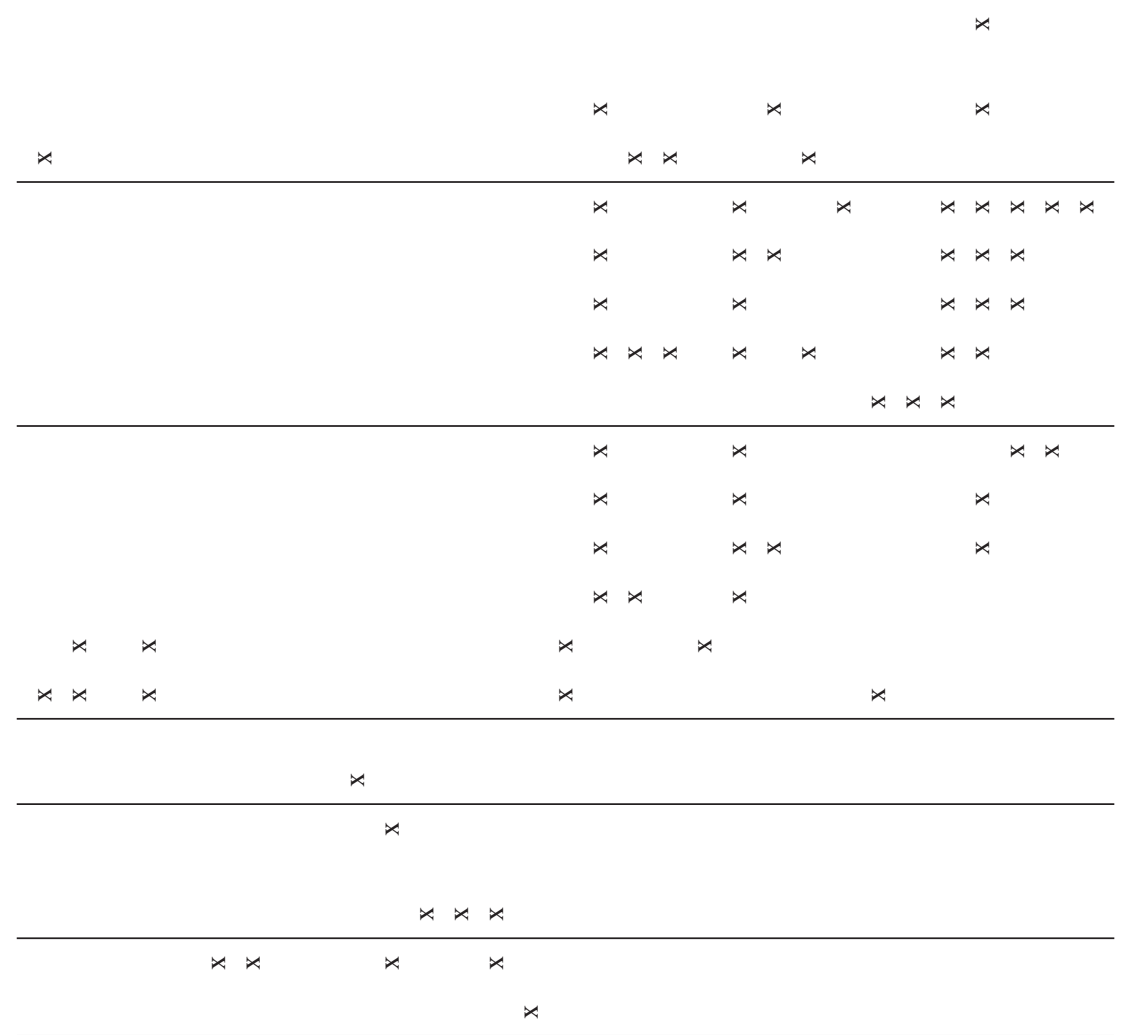

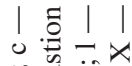
프를 응

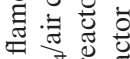
乙责要 我

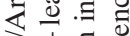

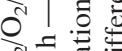
语荡 伺 I 宅艺言 어워 Z

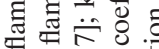
乙安考号 ते 空焉 ते ż山्: if on $\exists$ 异莳氞

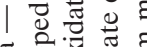

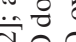
그은 $x$ 든. ठ 或

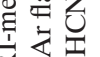
号完|

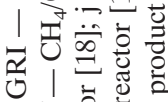
4 눈

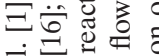
สं む 空

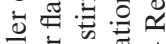

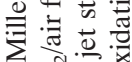
江.$\Xi$ 击当

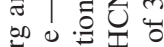
등

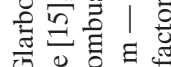

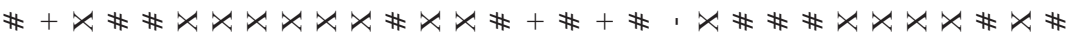

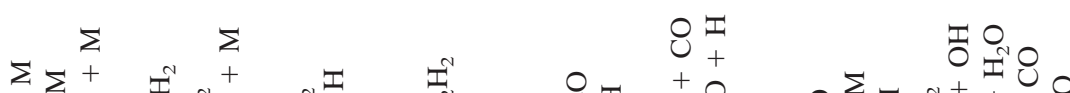

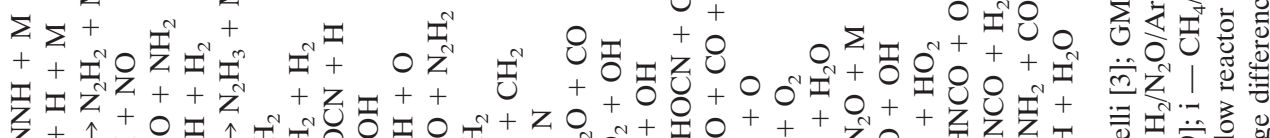

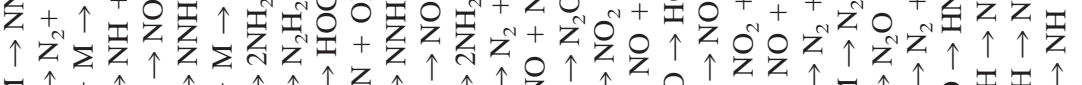

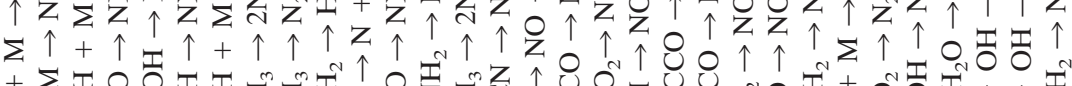

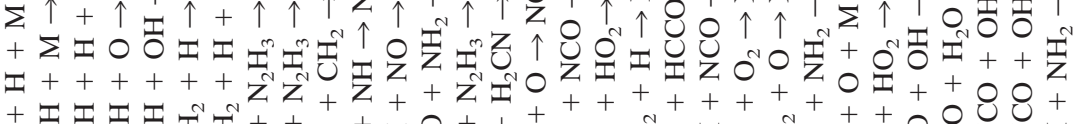

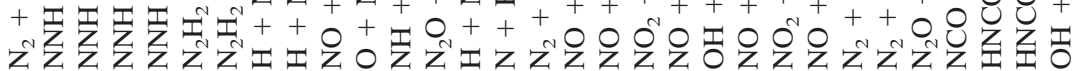

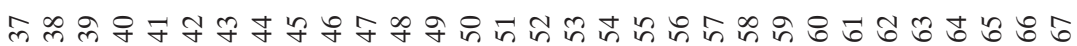
约芒芥

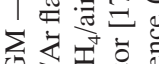

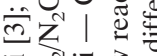
离

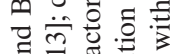
పี

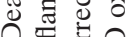

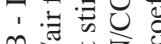

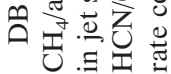


TABLE 2

Leeds NOx Reaction Mechanism, Rate Coefficients of Important Reactions of Nitrogenous Species

\begin{tabular}{|c|c|c|c|c|c|}
\hline & Reaction & $\mathrm{A} / \mathrm{cm}^{3} \mathrm{~mol}^{-1} \mathrm{~s}^{-1}$ & $\mathrm{n}$ & $\mathrm{E} / \mathrm{kJ} \mathrm{mol}^{-1}$ & Reference \\
\hline 1 & $\mathrm{O}_{2}+\mathrm{N} \rightarrow \mathrm{NO}+\mathrm{O}$ & $9.03 \times 10^{9}$ & 1.0 & 27.19 & {$[4]$} \\
\hline 2 & $\mathrm{~N}+\mathrm{OH} \rightarrow \mathrm{NO}+\mathrm{H}$ & $2.83 \times 10^{13}$ & 0 & 0 & {$[4]$} \\
\hline 3 & $\mathrm{NO}+\mathrm{N} \rightarrow \mathrm{N}_{2}+\mathrm{O}$ & $4.28 \times 10^{13}$ & 0 & 6.57 & {$[4]$} \\
\hline 4 & $\mathrm{NO}+\mathrm{NH} \rightarrow \mathrm{N}_{2} \mathrm{O}+\mathrm{H}$ & $4.16 \times 10^{14}$ & -0.45 & 0 & [25] \\
\hline 5 & $\mathrm{~N}_{2} \mathrm{O}+\mathrm{H} \rightarrow \mathrm{NO}+\mathrm{NH}$ & $9.92 \times 10^{20}$ & -1.57 & 150.25 & $\mathrm{a},[25]$ \\
\hline 6 & $\mathrm{NO}+\mathrm{NH} \rightarrow \mathrm{N}_{2}+\mathrm{OH}$ & $3.20 \times 10^{13}$ & 0 & 53.21 & {$[4],[26]$} \\
\hline 7 & $\mathrm{NO}+{ }^{1} \mathrm{CH}_{2} \rightarrow \mathrm{HCN}+\mathrm{OH}$ & $9.64 \times 10^{13}$ & 0 & 0 & [4] \\
\hline 8 & $\mathrm{O}+\mathrm{NCO} \rightarrow \mathrm{NO}+\mathrm{CO}$ & $3.16 \times 10^{13}$ & 0 & 0 & [27] \\
\hline \multirow[t]{2}{*}{9} & $\mathrm{HNO}+\mathrm{M} \rightarrow \mathrm{H}+\mathrm{NO}+\mathrm{M}$ & $5.09 \times 10^{16}$ & 0 & 203.69 & [28] \\
\hline & enhanced $3^{\text {rd }}$. body efficienc & ${ }_{4} / 3.0 / \mathrm{H}_{2} \mathrm{O} / 6.5 / \mathrm{CO}$ & $\mathrm{CO} / 0.75$ & t/ $\mathrm{N}_{2} / 0.4 / \mathrm{Ar} /$ & \\
\hline 10 & $\mathrm{O}+\mathrm{NH} \rightarrow \mathrm{NO}+\mathrm{H}$ & $5.50 \times 10^{13}$ & 0 & 0 & [29] \\
\hline 11 & $\mathrm{H}+\mathrm{NH} \rightarrow \mathrm{N}+\mathrm{H}_{2}$ & $1.02 \times 10^{13}$ & 0 & 0 & [4] \\
\hline \multirow[t]{2}{*}{12} & $\mathrm{NH}_{2}+\mathrm{NH}+\mathrm{M} \rightarrow \mathrm{N}_{2} \mathrm{H}_{3}+\mathrm{M}$ & $5.82 \times 10^{6}$ & 2.01 & -229.46 & $\mathrm{a},[26]$ \\
\hline & enhanced $3^{\text {rd }}$. body efficienc & ${ }_{4} / 3.0 / \mathrm{H}_{2} \mathrm{O} / 6.5 / \mathrm{CO}$ & $\mathrm{CO} / 0.75 /$ & $4 / \mathrm{N}_{2} / 0.4 / \mathrm{Ar} /$ & \\
\hline 13 & $\mathrm{NH}+\mathrm{H}_{2} \rightarrow \mathrm{H}+\mathrm{NH}_{2}$ & $1.77 \times 10^{11}$ & 0.38 & 47.98 & $\mathrm{a},[4]$ \\
\hline 14 & $\mathrm{OH}+\mathrm{NH} \rightarrow \mathrm{HNO}+\mathrm{H}$ & $1.00 \times 10^{12}$ & 0.3 & 8.314 & [29] \\
\hline 15 & $\mathrm{OH}+\mathrm{NH} \rightarrow \mathrm{N}+\mathrm{H}_{2} \mathrm{O}$ & $5.01 \times 10^{11}$ & 0.5 & 8.314 & [29] \\
\hline 16 & $2 \mathrm{NH} \rightarrow \mathrm{N}_{2}+2 \mathrm{H}$ & $5.13 \times 10^{13}$ & 0 & 0 & [26] \\
\hline 17 & $\mathrm{H}+\mathrm{NCO} \rightarrow \mathrm{NH}+\mathrm{CO}$ & $5.24 \times 10^{13}$ & 0 & 0 & [4] \\
\hline 18 & $\mathrm{HCN}+\mathrm{O} \rightarrow \mathrm{NCO}+\mathrm{H}$ & $8.45 \times 10^{5}$ & 2.1 & 25.57 & {$[4]$} \\
\hline 19 & $\mathrm{HCN}+\mathrm{O} \rightarrow \mathrm{NH}+\mathrm{CO}$ & $3.19 \times 10^{5}$ & 2.1 & 25.57 & [4] \\
\hline 20 & $\mathrm{HCN}+\mathrm{O} \rightarrow \mathrm{CN}+\mathrm{OH}$ & $2.22 \times 10^{5}$ & 2.1 & 25.57 & {$[4]$} \\
\hline 21 & $\mathrm{CN}+\mathrm{OH} \rightarrow \mathrm{HCN}+\mathrm{O}$ & $2.33 \times 10^{1}$ & 2.97 & -70.0 & $\mathrm{a},[4]$ \\
\hline 22 & $\mathrm{HCN}+\mathrm{OH} \rightarrow \mathrm{CN}+\mathrm{H}_{2} \mathrm{O}$ & $9.03 \times 10^{12}$ & 0.00 & 44.90 & [4] \\
\hline 23 & $\mathrm{CN}+\mathrm{H}_{2} \mathrm{O} \rightarrow \mathrm{HCN}+\mathrm{OH}$ & $9.35 \times 10^{10}$ & 0.6 & 23.47 & $\mathrm{a},[4]$ \\
\hline 24 & $\mathrm{HCN}+\mathrm{H} \rightarrow \mathrm{H}_{2}+\mathrm{CN}$ & $1.27 \times 10^{8}$ & 1.95 & 95.06 & $\mathrm{a},[4]$ \\
\hline 25 & $\mathrm{H}_{2}+\mathrm{CN} \rightarrow \mathrm{HCN}+\mathrm{H}$ & $1.93 \times 10^{4}$ & 2.87 & 6.82 & [4] \\
\hline \multirow[t]{2}{*}{26} & $\mathrm{HCN}+\mathrm{H}+\mathrm{M} \rightarrow \mathrm{H}_{2} \mathrm{CN}+\mathrm{M}$ & $4.89 \times 10^{12}$ & 0.54 & -17.06 & $\mathrm{a},[30]$ \\
\hline & enhanced $3^{\text {rd }}$. body efficienc & ${ }_{4} / 3.0 / \mathrm{H}_{2} \mathrm{O} / 6.5 / \mathrm{CO}$ & $\mathrm{CO} / 0.75 /$ & $4 / \mathrm{N}_{2} / 0.4 / \mathrm{Ar} /$ & \\
\hline \multirow[t]{2}{*}{27} & $\mathrm{H}_{2} \mathrm{CN}+\mathrm{M} \rightarrow \mathrm{HCN}+\mathrm{H}+\mathrm{M}$ & $7.50 \times 10^{14}$ & 0 & 92.05 & {$[30]$} \\
\hline & enhanced $3^{\text {rd }}$. body efficienc & ${ }_{4} / 3.0 / \mathrm{H}_{2} \mathrm{O} / 6.5 / \mathrm{CO}$ & $\mathrm{CO} / 0.75$ & $4 / \mathrm{N}_{2} / 0.4 / \mathrm{Ar} /$ & \\
\hline 28 & $\mathrm{~N}_{2}+\mathrm{CH} \rightarrow \mathrm{HCN}+\mathrm{N}$ & $1.57 \times 10^{12}$ & 0 & 75.08 & {$[4]$} \\
\hline 29 & $\mathrm{O}_{2}+\mathrm{CN} \rightarrow \mathrm{NCO}+\mathrm{O}$ & $7.23 \times 10^{12}$ & 0 & -1.75 & [4] \\
\hline 30 & $\mathrm{OH}+\mathrm{CN} \rightarrow \mathrm{NCO}+\mathrm{H}$ & $6.02 \times 10^{13}$ & 0 & 0 & [4] \\
\hline 31 & $\mathrm{CH}_{4}+\mathrm{CN} \rightarrow \mathrm{HCN}+\mathrm{CH}_{3}$ & $9.03 \times 10^{4}$ & 2.64 & -1.25 & [4] \\
\hline 32 & $\mathrm{~N}_{2} \mathrm{O}+\mathrm{H} \rightarrow \mathrm{N}_{2}+\mathrm{OH}$ & $4.40 \times 10^{14}$ & 0 & 78.99 & [31] \\
\hline 33 & $\mathrm{~N}_{2} \mathrm{O}+\mathrm{NH} \rightarrow \mathrm{HNO}+\mathrm{N}_{2}$ & $2.00 \times 10^{12}$ & 0 & 24.94 & [26] \\
\hline \multirow[t]{2}{*}{34} & $\mathrm{~N}_{2} \mathrm{O}+\mathrm{M} \rightarrow \mathrm{N}_{2}+\mathrm{O}+\mathrm{M}$ & $6.45 \times 10^{15}$ & 0 & 253 & [32] \\
\hline & enhanced $3^{\text {rd }}$. body efficienc & ${ }_{4} / 3.0 / \mathrm{H}_{2} \mathrm{O} / 6.5 / \mathrm{CO}$ & $\mathrm{CO} / 0.75$ & $4 / \mathrm{N}_{2} / 0.4 / \mathrm{Ar} /$ & \\
\hline \multirow[t]{4}{*}{35} & $\mathrm{NH}_{2}+\mathrm{H}+\mathrm{M} \rightarrow \mathrm{NH}_{3}+\mathrm{M}$ & $4.20 \times 10^{11}$ & 0.78 & -3.02 & $\mathrm{a},[4]$ \\
\hline & low pressure limit: & $2.26 \times 10^{11}$ & 0.78 & -116.18 & \\
\hline & Troe parameters: 0.424581102 & & & & \\
\hline & enhanced $3^{\text {rd }}$. body efficienc & ${ }_{4} / 3.0 / \mathrm{H}_{2} \mathrm{O} / 6.5 / \mathrm{CO}$ & $\mathrm{CO} / 0.75 /$ & $4 / \mathrm{N}_{2} / 0.4 / \mathrm{Ar} /$ & \\
\hline 36 & $\mathrm{O}+\mathrm{NH}_{2} \rightarrow \mathrm{HNO}+\mathrm{H}$ & $8.93 \times 10^{14}$ & -0.489 & 1.36 & [33] \\
\hline \multirow[t]{3}{*}{37} & $\mathrm{~N}_{2}+\mathrm{H}+\mathrm{M} \rightarrow \mathrm{NNH}+\mathrm{M}$ & $4.24 \times 10^{11}$ & 1.13 & 35.99 & $a,[34]$ \\
\hline & enhanced $3^{\text {rd }}$. body efficienc & ${ }_{4} / 3.0 / \mathrm{H}_{2} \mathrm{O} / 6.5 / \mathrm{CO}$ & $\mathrm{CO} / 0.75 /$ & $4 / \mathrm{N}_{2} / 0.4 / \mathrm{Ar} /$ & \\
\hline & $\mathrm{N}_{2}+\mathrm{H} \rightarrow \mathrm{NNH}$ & $5.09 \times 10^{6}$ & 0.63 & 23.19 & $\mathrm{a},[34]$ \\
\hline \multirow[t]{3}{*}{38} & $\mathrm{NNH}+\mathrm{M} \rightarrow \mathrm{N}_{2}+\mathrm{H}+\mathrm{M}$ & $2.50 \times 10^{13}$ & 0.5 & 12.8 & {$[34]$} \\
\hline & enhanced $3^{\text {rd }}$. body efficienc & ${ }_{4} / 3.0 / \mathrm{H}_{2} \mathrm{O} / 6.5 / \mathrm{CO}$ & $\mathrm{CO} / 0.75 /$ & $4 / \mathrm{N}_{2} / 0.4 / \mathrm{Ar}$ & \\
\hline & $\mathrm{NNH} \rightarrow \mathrm{N}_{2}+\mathrm{H}$ & $3.00 \times 10^{8}$ & 0 & 0 & [34] \\
\hline \multirow[t]{2}{*}{39} & $\mathrm{NNH}+\mathrm{H}+\mathrm{M} \rightarrow \mathrm{N}_{2} \mathrm{H}_{2}+\mathrm{M}$ & $6.11 \times 10^{11}$ & 0.81 & -50.07 & $\mathrm{a},[26]$ \\
\hline & enhanced $3^{\text {rd }}$. body efficienc & ${ }_{4} / 3.0 / \mathrm{H}_{2} \mathrm{O} / 6.5 / \mathrm{CO}$ & $\mathrm{CO} / 0.75$ & $4 / \mathrm{N}_{2} / 0.4 / \mathrm{Ar} /$ & \\
\hline 40 & $\mathrm{NNH}+\mathrm{O} \rightarrow \mathrm{NH}+\mathrm{NO}$ & $1.65 \times 10^{14}$ & -0.23 & -4.24 & $b,[34]$ \\
\hline 41 & $\mathrm{NNH}+\mathrm{OH} \rightarrow \mathrm{NO}+\mathrm{NH}_{2}$ & $2.24 \times 10^{15}$ & -1.25 & -4.73 & $\mathrm{a},[35],[36]$ \\
\hline 42 & $\mathrm{~N}_{2} \mathrm{H}_{2}+\mathrm{H} \rightarrow \mathrm{NNH}+\mathrm{H}_{2}$ & $1.00 \times 10^{13}$ & 0 & 4.16 & [26] \\
\hline
\end{tabular}


TABLE 2

Leeds NOx Reaction Mechanism, Rate Coefficients of Important Reactions of Nitrogenous Species

\begin{tabular}{|c|c|c|c|c|c|}
\hline & Reaction & $\mathrm{A} / \mathrm{cm}^{3} \mathrm{~mol}^{-1} \mathrm{~s}^{-1}$ & $\mathrm{n}$ & $\mathrm{E} / \mathrm{kJ} \mathrm{mol}{ }^{-1}$ & Reference \\
\hline 43 & \multicolumn{5}{|c|}{ enhanced $3^{\mathrm{rd}}$. body efficiencies: $\mathrm{CH}_{4} / 3.0 / \mathrm{H}_{2} \mathrm{O} / 6.5 / \mathrm{CO}_{2} / 1.5 / \mathrm{CO} / 0.75 / \mathrm{O}_{2} / 0.4 / \mathrm{N}_{2} / 0.4 / \mathrm{Ar} / 0.35 /$} \\
\hline 44 & $\mathrm{H}+\mathrm{N}_{2} \mathrm{H}_{3} \rightarrow 2 \mathrm{NH}_{2}$ & $1.58 \times 10^{12}$ & 0 & 0 & [26] \\
\hline 45 & $\mathrm{H}+\mathrm{N}_{2} \mathrm{H}_{3} \rightarrow \mathrm{N}_{2} \mathrm{H}_{2}+\mathrm{H}_{2}$ & $1.00 \times 10^{12}$ & 0 & 8.314 & [29] \\
\hline 46 & $\mathrm{NO}+\mathrm{CH}_{2} \rightarrow \mathrm{HOCN}+\mathrm{H}$ & $1.39 \times 10^{12}$ & 0 & -4.6 & $\mathrm{c},[30]$ \\
\hline 47 & $\mathrm{O}+\mathrm{NH} \rightarrow \mathrm{N}+\mathrm{OH}$ & $3.72 \times 10^{13}$ & 0 & 0 & [29] \\
\hline 48 & $\mathrm{NH}+\mathrm{NO} \rightarrow \mathrm{NNH}+\mathrm{O}$ & $3.60 \times 10^{12}$ & 0.18 & 41.15 & $\mathrm{a},[30]$ \\
\hline 49 & $\mathrm{~N}_{2} \mathrm{O}+\mathrm{NH}_{2} \rightarrow \mathrm{NO}+\mathrm{N}_{2} \mathrm{H}_{2}$ & $1.45 \times 10^{13}$ & -0.06 & 28.49 & $\mathrm{a},[26]$ \\
\hline 50 & $\mathrm{H}+\mathrm{N}_{2} \mathrm{H}_{3} \rightarrow 2 \mathrm{NH}_{2}$ & $1.58 \times 10^{12}$ & 0 & 0 & [26] \\
\hline 51 & $\mathrm{~N}+\mathrm{H}_{2} \mathrm{CN} \rightarrow \mathrm{N}_{2}+\mathrm{CH}_{2}$ & $2.00 \times 10^{13}$ & 0 & 0 & {$[30]$} \\
\hline 52 & $\mathrm{~N}_{2}+\mathrm{O} \rightarrow \mathrm{NO}+\mathrm{N}$ & $1.81 \times 10^{14}$ & 0 & 318.43 & [4] \\
\hline 53 & $\mathrm{NO}+\mathrm{NCO} \rightarrow \mathrm{N}_{2} \mathrm{O}+\mathrm{CO}$ & $1.39 \times 10^{18}$ & -1.73 & 3.16 & [4] \\
\hline 54 & $\mathrm{NO}+\mathrm{HO}_{2} \rightarrow \mathrm{NO}_{2}+\mathrm{OH}$ & $2.09 \times 10^{12}$ & 0 & -2.00 & [28] \\
\hline 55 & $\mathrm{NO}_{2}+\mathrm{H} \rightarrow \mathrm{NO}+\mathrm{OH}$ & $3.47 \times 10^{14}$ & 0 & 6.15 & {$[26]$} \\
\hline 56 & $\mathrm{NO}+\mathrm{HCCO} \rightarrow \mathrm{HOCN}+\mathrm{CO}$ & $2.00 \times 10^{13}$ & 0 & 0 & $\mathrm{c},[30]$ \\
\hline 57 & $\mathrm{OH}+\mathrm{NCO} \rightarrow \mathrm{NO}+\mathrm{CO}+\mathrm{H}$ & $1.00 \times 10^{13}$ & 0 & 0 & {$[30]$} \\
\hline 58 & $\mathrm{NO}+\mathrm{O}_{2} \rightarrow \mathrm{NO}_{2}+\mathrm{O}$ & $1.04 \times 10^{11}$ & 0.41 & 193.57 & $\mathrm{a},[30]$ \\
\hline 59 & $\mathrm{NO}_{2}+\mathrm{O} \rightarrow \mathrm{NO}+\mathrm{O}_{2}$ & $1.00 \times 10^{13}$ & 0 & 2.51 & {$[30]$} \\
\hline 60 & $\mathrm{NO}+\mathrm{NH}_{2} \rightarrow \mathrm{N}_{2}+\mathrm{H}_{2} \mathrm{O}$ & $5.48 \times 10^{15}$ & -1.71 & 0 & {$[35],[36]$} \\
\hline 61 & $\mathrm{~N}_{2}+\mathrm{O}+\mathrm{M} \rightarrow \mathrm{N}_{2} \mathrm{O}+\mathrm{M}$ & $9.31 \times 10^{8}$ & 1.34 & 78.55 & $\mathrm{a},[32]$ \\
\hline & enhanced $3^{\text {rd }}$. body efficien & $\mathrm{H}_{4} / 3.0 / \mathrm{H}_{2} \mathrm{O} / 6.5 / \mathrm{C}$ & $\mathrm{CO} / 0.7$ & $0.4 / \mathrm{N}_{2} \mathrm{Ar} / 0.3$ & \\
\hline 62 & $\mathrm{~N}_{2}+\mathrm{HO}_{2} \rightarrow \mathrm{N}_{2} \mathrm{O}+\mathrm{OH}$ & $4.37 \times 10^{9}$ & 0.48 & 152.44 & $\mathrm{a},[37]$ \\
\hline 63 & $\mathrm{~N}_{2} \mathrm{O}+\mathrm{OH} \rightarrow \mathrm{N}_{2}+\mathrm{HO}_{2}$ & $6.31 \times 10^{11}$ & 0 & 41.57 & [37] \\
\hline 64 & $\mathrm{NCO}+\mathrm{H}_{2} \mathrm{O} \rightarrow \mathrm{HNCO}+\mathrm{OH}$ & $4.71 \times 10^{12}$ & 0.04 & 55.25 & $\mathrm{a},[38]$ \\
\hline 65 & $\mathrm{HNCO}+\mathrm{OH} \rightarrow \mathrm{NCO}+\mathrm{H}_{2} \mathrm{O}$ & $1.99 \times 10^{12}$ & 0 & 23.18 & [38] \\
\hline 66 & $\mathrm{HNCO}+\mathrm{OH} \rightarrow \mathrm{NH}_{2}+\mathrm{CO}_{2}$ & $1.99 \times 10^{12}$ & 0 & 23.18 & [38] \\
\hline 67 & $\mathrm{OH}+\mathrm{NH}_{2} \rightarrow \mathrm{NH}+\mathrm{H}_{2} \mathrm{O}$ & $5.01 \times 10^{11}$ & 0.5 & 8.314 & [28] \\
\hline
\end{tabular}

a - derived from the reverse reaction and thermodynamics; $\mathrm{b}$ - adjusted to half the value recommended by Bozzelli \& Dean [34] to improve the fit to the data of Harrington et al. [16]; c - for the HCNO product channel.

\section{Reactions of NO}

Reaction 7, $\left(\mathrm{NO}+{ }^{1} \mathrm{CH}_{2} \rightarrow \mathrm{HCN}+\mathrm{OH}\right)$ is the most important reaction in this group, because

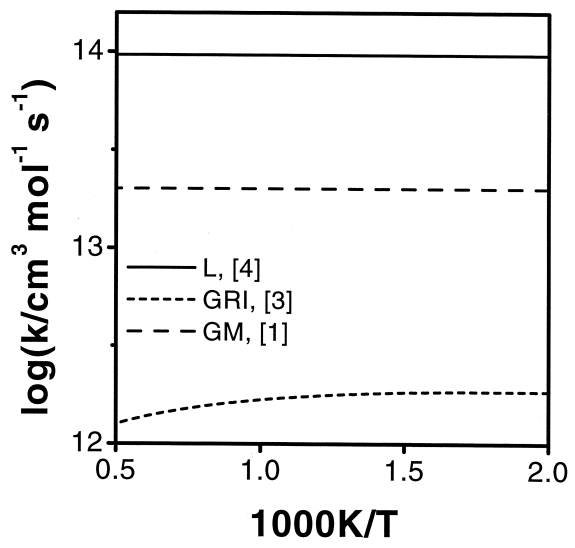

Fig. 9. Temperature dependence of the rate coefficient of reaction 7: $\mathrm{NO}+{ }^{1} \mathrm{CH}_{2} \rightarrow \mathrm{HCN}+\mathrm{OH}$. it has a high sensitivity for a variety of nitrogencontaining species in all types of experiment. The temperature dependence of the rate coefficient is shown in Fig. 9, and shows large differences between the mechanisms. The rate coefficient used in the Leeds NOx mechanism for this reaction is from the CEC evaluation [4] of the overall rate coefficient for all product channels, and therefore represents an upper limit. Dean and Bozzelli [3] only include reactions of ${ }^{3} \mathrm{CH}_{2}$ in their mechanism. Reaction of ${ }^{1} \mathrm{CH}_{2}$ with $\mathrm{N}_{2}$ was considered [3], but deemed to be unimportant. ${ }^{1} \mathrm{CH}_{2}$ is produced in several reactions in the oxidation of hydrocarbons. Its reactivity is often significantly higher than that of ${ }^{3} \mathrm{CH}_{2}$, so its reactions should be included. GRI-mech 3.0 [2] uses Dean and Bozzelli's [3] rate parameters for both ${ }^{1} \mathrm{CH}_{2}$ and ${ }^{3} \mathrm{CH}_{2}$, arguing that because a reaction is exothermic, its rate parameters for ${ }^{1} \mathrm{CH}_{2}$ and ${ }^{3} \mathrm{CH}_{2}$ are identi- 


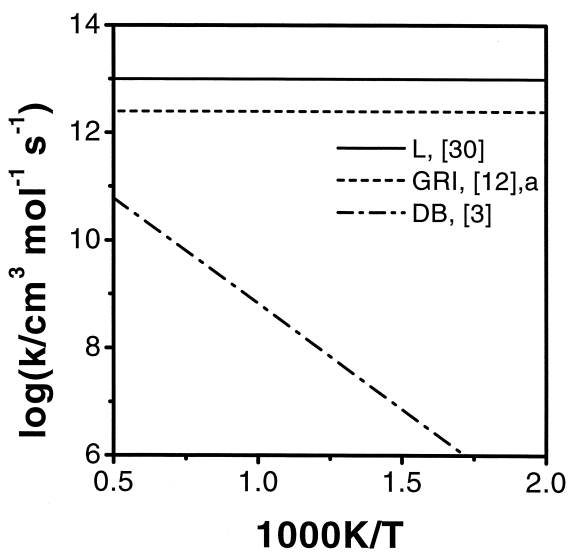

Fig. 10. Temperature dependence of the rate coefficient of reaction 57: $\mathrm{OH}+\mathrm{NCO} \rightarrow \mathrm{NO}+\mathrm{CO}+\mathrm{H}$; a-Altered in the optimization process performed to generate the GRI mechanism.

cal. Glarborg and Miller [1] use an estimated value of the rate parameters for this reaction.

Reaction 57, $(\mathrm{OH}+\mathrm{NCO} \rightarrow \mathrm{NO}+\mathrm{CO}+\mathrm{H})$ is important in controlling $\mathrm{NO}$ and $\mathrm{N}_{2} \mathrm{O}$ in the flow tube experiments of Glarborg and Miller [17] and the temperature dependence of its rate coefficient is shown in Fig. 10. No experimental determinations have been made of this reaction's rate coefficient. The rate coefficient used in both the Leeds NOx mechanism and GRImech 3.0 was obtained from an estimate of Miller et al. [12], although in GRI-mech 3.0 it has been reduced by a factor of four by their optimization process. Dean and Bozzelli [3] consider several possible product channels for this reaction. From QRRK calculations they obtained a rate coefficient that is much lower and has strong temperature dependence, to the extent that this product channel accounts for less than $1 \%$ of the reaction up to $2000 \mathrm{~K}$. Glarborg and Miller [1] do not include this product channel, but assume that the products are $\mathrm{NO}+\mathrm{HCO}$, for which they use a rate coefficient, estimated as an upper limit by Miller and Bowman [38], and similar to Dean and Bozzelli's [3] QRRK calculation.

Reactions 6 and 49, (NO $+\mathrm{NH} \rightarrow \mathrm{N}_{2}+\mathrm{OH}$ and $\mathrm{N}_{2} \mathrm{O}+\mathrm{NH}_{2} \rightarrow \mathrm{NO}+\mathrm{N}_{2} \mathrm{H}_{2}$ ) are of minor importance, only being significant in the low pressure $\mathrm{H}_{2} / \mathrm{N}_{2} \mathrm{O} / \mathrm{Ar}$ flame. The rate expression used in the Leeds NOx mechanism for reaction 6 is based on the work of Mertens et al. [26]

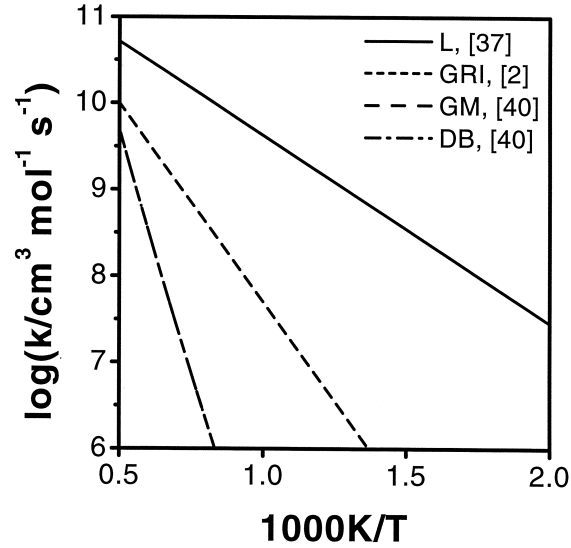

Fig. 11. Temperature dependence of the rate coefficient of reaction 63: $\mathrm{N}_{2} \mathrm{O}+\mathrm{OH} \rightarrow \mathrm{N}_{2}+\mathrm{HO}_{2}$.

using a shock tube. All the other rate parameters used for reactions 6 and 49 are estimates, either derived from QRRK calculations [3] or fitted to theoretical calculations [25]. In the case of GRI-mech 3.0, $\mathrm{N}_{2} \mathrm{H}_{2}$ was not included in the mechanism, so that reaction 49 was absent.

\section{Reactions of $\mathrm{N}_{2} \mathrm{O}$}

Reaction 32, $\left(\mathrm{N}_{2} \mathrm{O}+\mathrm{H} \rightarrow \mathrm{N}_{2}+\mathrm{OH}\right)$ is important in each type of experiment investigated. The rate parameters used in all mechanisms are derived from the experimental measurements of Fontijn et al. [31], except for Dean and Bozzelli [3], who used the more recent measurements of Fontijn et al. [39]. The major difference is at low temperature, where the rate expression used by Glarborg and Miller [1] deviates from that used by the other mechanisms. This difference arises because they used the original expression proposed by Fontijn et al. [31] that is made up of both a high temperature and low temperature component. The reaction is only important at higher temperatures and so the difference is not expected to have any significant impact on the simulations.

Reaction 63, $\left(\mathrm{N}_{2} \mathrm{O}+\mathrm{OH} \rightarrow \mathrm{N}_{2}+\mathrm{HO}_{2}\right)$ is important for both the jet-stirred reactor and flow tube experiments. The temperature dependence of its rate coefficient is shown in Fig. 11, showing very large differences between the different mechanisms. The Leeds NOx mechanism uses rate parameters applicable at high temperatures [37]. The mechanisms of Dean and Boz- 
zelli [3] and Glarborg and Miller [1] both use a rate coefficient calculated by Mebel et al. [40] from transition state theory; it is much lower than either the Leeds NOx mechanism or GRImech 3.0. The rate coefficient in GRI-mech 3.0 [2] is intermediate between the values used in the mechanisms of Leeds NOx mechanism and those of Dean and Bozzelli [3] and Glarborg and Miller [1]. The only high temperature experimental measurement at $1123 \mathrm{~K}$ [41] is consistent with the rate coefficient in GRI-mech 3.0 suggesting that the rate parameter expressions used in the Leeds NOx mechanism, and those of Dean and Bozzelli, and Glarborg and Miller may need to be adjusted, although clearly the reaction is poorly characterized.

Reactions 61 and 62, $\left(\mathrm{N}_{2}+\mathrm{O}+\mathrm{M} \rightarrow \mathrm{N}_{2} \mathrm{O}+\right.$ $\mathrm{M}$ and $\left.\mathrm{N}_{2}+\mathrm{HO}_{2} \rightarrow \mathrm{N}_{2} \mathrm{O}+\mathrm{OH}\right)$ are less important, only being significant for a jet-stirred reactor. For reaction 62 , the rate coefficient expressions in all four mechanisms derive from experimental measurements of the reverse reaction, combined with thermodynamics to calculate the forward reaction rate parameters. The Leeds NOx mechanism uses an expression based on earlier data [32] over a narrower temperature range than the other three mechanisms and, given the excellent agreement between subsequent experimental measurements, should be updated. The treatment of this reaction in the Leeds NOx mechanism exemplifies the limitations of an adherence to evaluated rate data. In all four mechanisms, the rate coefficient for reaction 62 was obtained from thermodynamics and the reverse reaction, 63, discussed previously. The limitations in our understanding of reaction 63 clearly also apply to reaction 62 .

\section{Reactions of $\mathrm{HCN}$}

Reaction $22,\left(\mathrm{HCN}+\mathrm{OH} \rightarrow \mathrm{CN}+\mathrm{H}_{2} \mathrm{O}\right)$ is the most important reaction involving $\mathrm{HCN}$ and is significant in all types of experiment. Figure 12 shows the temperature dependence of it's rate coefficient. The Leeds NOx mechanism uses the rate coefficient as recommended in the latest CEC evaluation [4]. Glarborg and Miller [1] and Dean and Bozzelli [3] use the rate coefficient measured by Woolridge et al. [43] from monitoring $\mathrm{OH}$ and $\mathrm{CN}$ in a shock tube study of this

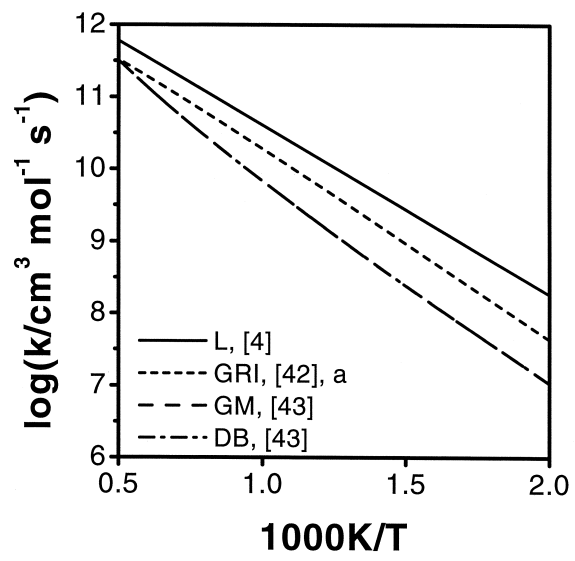

Fig. 12. Temperature dependence of the rate coefficient of reaction 22: $\mathrm{HCN}+\mathrm{OH} \rightarrow \mathrm{CN}+\mathrm{H}_{2} \mathrm{O}$; a-Calculated from the reverse reaction and thermodynamics.

reaction. GRI-mech 3.0 [2] uses rate coefficients for this reaction that have been obtained from thermodynamics and Jacobs et al. [42], who used flash photolysis combined with laser induced fluorescence to measure the rate coefficient of the reverse reaction between 518 to $1027 \mathrm{~K}$.

Reaction 28, $\left(\mathrm{N}_{2}+\mathrm{CH} \rightarrow \mathrm{HCN}+\mathrm{N}\right)$. In the Leeds NOx mechanism the rate coefficient for this reaction was obtained from the latest CEC evaluation [4] of the overall rate, in which this channel was assumed to dominate at high temperatures. The other three mechanisms use alternative rate parameters, based on experimental measurements in GRI-mech 3.0 [2] and Dean and Bozzelli [3], and theoretical calculation in Glarborg and Miller [1].

Reactions 26 and 27, $(\mathrm{HCN}+\mathrm{H}+\mathrm{M}=$ $\left.\mathrm{H}_{2} \mathrm{CN}+\mathrm{M}\right)$. No measurements exist for this reaction and all four mechanisms use estimated rate parameters from various sources.

\section{Reactions of NH}

Reaction $14,(\mathrm{OH}+\mathrm{NH} \rightarrow \mathrm{HNO}+\mathrm{H})$ is the most significant reaction for $\mathrm{NH}$, and is important in three of the laminar flame simulations. In the absence of experimental measurements, all four mechanisms use estimated rate parameters for this reaction, the temperature dependence of which is shown in Fig. 13. The Leeds NOx mechanism uses a rate coefficient estimated by Hanson and Salimian [28], who sug- 


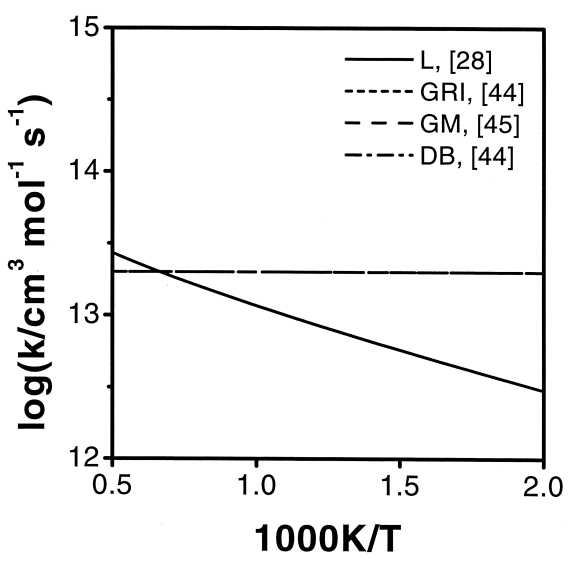

Fig. 13. Temperature dependence of the rate coefficient of reaction 14: $\mathrm{OH}+\mathrm{NH} \rightarrow \mathrm{HNO}+\mathrm{H}$.

gested it to be applicable for temperatures above $1000 \mathrm{~K}$. Figure 13 shows that at these temperatures, the difference between the Leeds NOx mechanism and the other three mechanisms, who all use the same temperature independent value estimated by analogy with similar reactions, is minor.

Reactions 15 and $47\left(\mathrm{OH}+\mathrm{NH} \rightarrow \mathrm{N}+\mathrm{H}_{2} \mathrm{O}\right.$ and $\mathrm{O}+\mathrm{NH} \rightarrow \mathrm{N}+\mathrm{OH}$ ) are important in two of the laminar flame simulations. There are no experimental measurements for reaction 15, and all four mechanisms use estimated rate parameters. The main difference arises in Dean and Bozzelli's mechanism [3], which uses an estimation procedure for radical-radical hydrogen atom transfer to obtain the rate parameters. For reaction 47 there are major differences between the four mechanisms, as can be observed in Fig. 14, which shows the temperature dependence of the rate coefficient. GRI-mech 3.0 [2], and Glarborg and Miller [1] do not include this product channel. Dean and Bozzelli [3] again use their radical-radical hydrogen transfer estimation to obtain rate parameters, and comment that they expect this product channel to be a minor component of the overall reaction. A much faster rate coefficient is used in the Leeds NOx mechanism, based on the shock tube experiments of Mertens et al. [26] who measured the overall rate of reaction, and proposed product branching ratios that make this channel important.

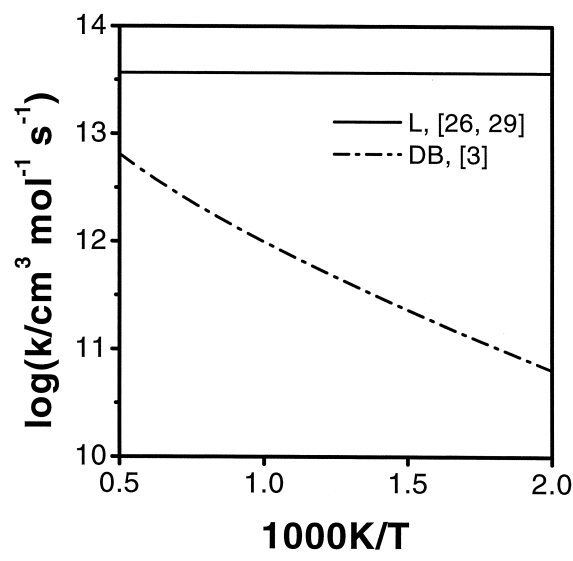

Fig. 14. Temperature dependence of the rate coefficient of reaction 47: $\mathrm{O}+\mathrm{NH} \rightarrow \mathrm{N}+\mathrm{OH}$.

\section{Reactions of NNH}

Reaction $38\left(\mathrm{NNH}+\mathrm{M} \rightarrow \mathrm{N}_{2}+\mathrm{H}+\mathrm{M}\right)$. The temperature dependence of the rate coefficient is shown in Fig. 15. Both the Leeds NOx mechanism and Dean and Bozzelli's [3] use the same value published by Dean and Bozzelli [34], who concluded that $\mathrm{NNH}$ dissociation is dominated by tunneling, and derived a two term rate coefficient consisting of pressure-dependent and pressure-independent terms. The GRImech 3.0 [2] employs a similar expression. However, there is some confusion in their choice of pressure-dependent parameters, which, if corrected, give an almost identical rate expression to that used by Dean and Bozzelli [3] and the

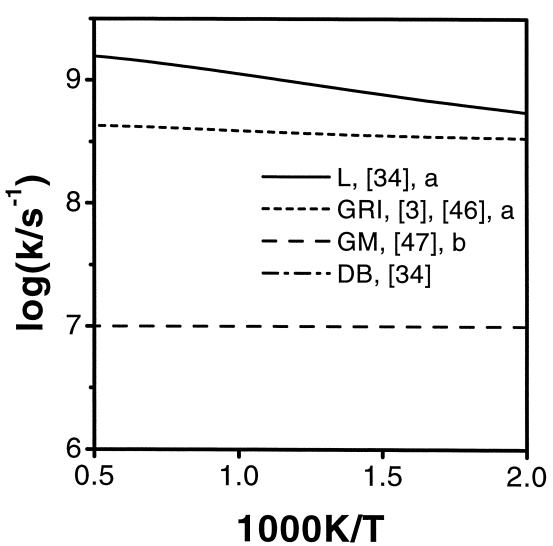

Fig. 15. Temperature dependence of the rate coefficient of reaction 38: $\mathrm{NNH}+\mathrm{M} \rightarrow \mathrm{N}_{2}+\mathrm{H}+\mathrm{M}$; a-Rate coefficient values a composite of pressure dependent and independent reactions; $b-$ Rate coefficient is independent of pressure. 
Leeds NOx mechanism. The confusion is that they reference Dean and Bozzelli [3], but instead of choosing the value they actually recommend, i.e., the one dominated by tunneling, they choose the value mentioned in the discussion of Dean and Bozzelli [3,34] who used a QRRK calculation to estimate rate parameters. In this calculation, Dean and Bozzelli [3,34] did some adjustment of barrier heights to try to simulate the effects of tunneling and obtained an expression about a factor of 20 lower than the tunneling expression. However, when combined with the pressure independent term for reaction 38, the overall difference is not so large. Glarborg and Miller [1] only use a pressure-independent rate coefficient obtained from their earlier work [47], in which the value was chosen to match their simulations of NO consumption to experimentally observed results in flow reactor experiments.

Reactions 39 and $42\left(\mathrm{NNH}+\mathrm{H}+\mathrm{M} \rightarrow \mathrm{N}_{2} \mathrm{H}_{2}\right.$ $+\mathrm{M}$ and $\mathrm{N}_{2} \mathrm{H}_{2}+\mathrm{H} \rightarrow \mathrm{NNH}+\mathrm{H}_{2}$ ) are absent from GRI-mech 3.0, as it does not include $\mathrm{N}_{2} \mathrm{H}_{2}$. The other three mechanisms all use estimates from various sources; the Leeds NOx mechanism uses data originating from Hanson and Salimian [28]. The rate parameters for reaction 39, $\mathrm{NNH}+\mathrm{H}+\mathrm{M} \rightarrow \mathrm{N}_{2} \mathrm{H}_{2}+\mathrm{M}$ obtained from thermodynamics and the reverse reaction, illustrate the dangers inherent in using estimated rates over a too wide range of conditions. If extrapolated to low temperatures, the Leeds NOx and Glarborg and Miller [1] mechanisms predict unreasonably fast rate coefficients.

\section{Quality of Simulations}

In many cases, agreement between the simulations and experimental measurements is good. The most notable exceptions are the predictions of [NO] in Fig. 5 for a rich hydrogen/air flame. This experiment was specifically designed to investigate a route forming $\mathrm{NO}$, involving the intermediate species NNH. The simulations of these experiments, while highlighting the importance of $\mathrm{NNH}$ under these conditions, show poor agreement for the [NO] profiles. This suggests that some optimization of the mechanism is required and that this route is not well understood, particularly given the discrepancy
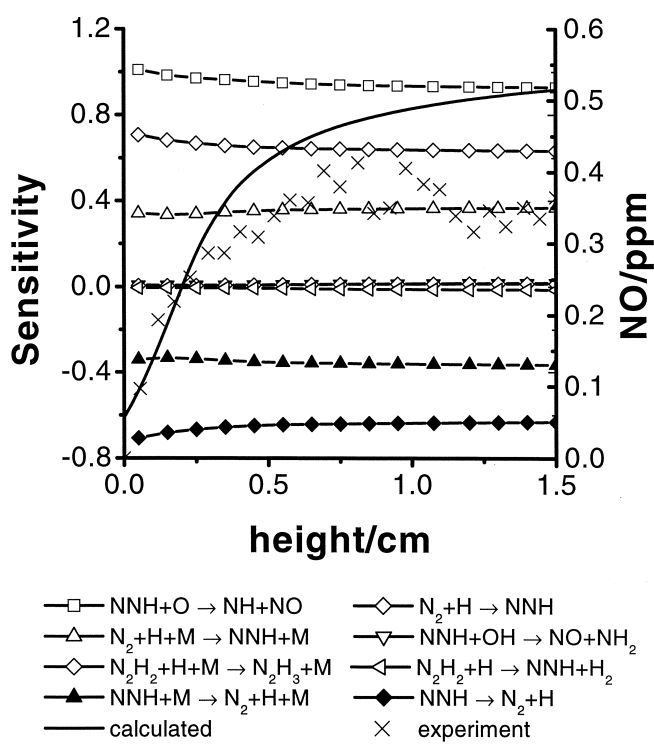

Fig. 16. [NO] sensitivities in a rich $(\Phi=1.5) \mathrm{H}_{2}$ /air flame [16] at 78 Torr as a function of height above the burner.

between the simulated and experimentally observed pressure dependence of [NO]. Figure 16 shows the sensitivities of [NO] with respect to important reactions as a function of height above the burner for the simulation at 78 Torr. Figure 16 demonstrates that the reaction involving nitrogenous species with the largest sensitivity for $\mathrm{NO}$ formation is reaction 40 : $\mathrm{NNH}+\mathrm{O}$ $\rightarrow \mathrm{NO}+\mathrm{NH}$, although Table 1 indicates that the differences in rate parameters between the mechanisms are not large for this reaction. The rate coefficient of reaction 40 has been adjusted to half the value proposed by Bozzelli and Dean [34] to improve the fit with measurements, although as can be seen in Fig. 5, the fit is still not as good as that achieved by GRI-mech 3.0 [2]. There is no obvious solution to the problem of simulating the observed pressure dependence of [NO] without drastic changes to reaction rate coefficients, or mechanistic alterations. Altering the rate of reaction 40 , while having the largest effect on [NO], will not affect the pressure dependence, because it has a similar sensitivity with respect to [NO] in the simulation at 38 Torr. Figure 16 shows that, apart from reaction 40 , the other important reactions have almost equal and opposite contributions from their reverse components. Therefore, changing their rate parameters will have little effect on the simulated $[\mathrm{NO}]$ profile, and the only possibility 
for effecting any change is to alter the thermodynamic data used for NNH. This conclusion was confirmed by further analysis of the simulated results, which show that reactions 37 and 38 are in equilibrium, with rates of reaction over four orders of magnitude greater than the rate of conversion of $\mathrm{NNH}$ to $\mathrm{NO}$ via reaction 40 .

In the simulations of the flow tube experiments of Glarborg and Miller [17], there is a tendency to overpredict the destruction of $\mathrm{HCN}$ at any given temperature, with subsequent distortions of the predictions of other nitrogencontaining species. This can be seen most clearly in Fig. 8a. From the concentration sensitivities, at $1100 \mathrm{~K}$ reaction $22: \mathrm{HCN}+\mathrm{OH} \rightarrow$ $\mathrm{CN}+\mathrm{H}_{2} \mathrm{O}$ is the dominant reaction controlling $\mathrm{HCN}, \mathrm{N}_{2} \mathrm{O}$ and NO. As can be seen in Fig. 12, and as discussed in 'Reactions of HCN', the Leeds NOx mechanism uses a rate coefficient for this reaction, which is much faster than those employed in the other mechanisms, especially by Dean and Bozzelli [3] and Glarborg and Miller [1]. Replacement of the rate coefficient used in the Leeds NOx mechanism with the more recent measurement of Woolridge et al. [43], as used in these mechanisms, will improve the quality of the Leeds NOx mechanism simulations of the flow tube data.

When simulating the measurements of Bartok et al. [18], the fuel-rich simulations show an underproduction of NO in the undoped experiment, but with $\mathrm{NO}$ added there is an excess consumption of NO. Under these conditions, there are only three reactions of nitrogenous species found to be important in controlling [NO]. These are reactions 28: $\mathrm{N}_{2}+\mathrm{CH} \rightarrow \mathrm{HCN}$ $+\mathrm{N}$, important in the undoped experiment, reaction 56: $\mathrm{NO}+\mathrm{HCCO} \rightarrow \mathrm{HOCN}+\mathrm{CO}$, important for both the doped and undoped experiments, and reaction 7: $\mathrm{NO}+{ }^{1} \mathrm{CH}_{2} \rightarrow$ $\mathrm{HCN}+\mathrm{OH}$, important in the doped experiment. Reaction 28, as discussed in 'Reactions of HCN', has a positive sensitivity with respect to $\mathrm{NO}$, and the rate coefficient is already at its upper limit, giving little scope for achieving an improvement in the simulations by its adjustment. Reaction 7, discussed in 'Reactions of NO', may be adjusted to improve the simulations. The rate coefficient used also represents an upper limit, which is considerably faster than that used in the other mechanisms. However, this reaction is only found to be important for simulating an experiment with $\mathrm{NO}$ as dopant. Reaction 56 is important for both the doped and undoped experiments, and here there is some scope for adjustment. This reaction is absent from the other three reaction mechanisms, which instead assume that the products are $\mathrm{HCNO}+\mathrm{CO}$, or $\mathrm{HCN}+\mathrm{CO}_{2}$. There is little published data on this reaction. There are two room temperature measurements of the overall rate coefficient of $1.3 \times 10^{13}$ [48] and $2.35 \times 10^{13}[49] \mathrm{cm}^{3} \mathrm{~mol}^{-1} \mathrm{~s}^{-1}$, respectively. Boullart et al. [50] measured the rate coefficient between 290 to $670 \mathrm{~K}$, using the technique of discharge flow with molecular beam mass spectrometry, and observed a slight temperature dependence. At $700 \mathrm{~K}$, they also measured the product branching ratio, obtaining $77 \%$ via the $\mathrm{CO}$ channel and $23 \%$ via the $\mathrm{CO}_{2}$ channel. Therefore, there is scope to adjust the rate coefficient and the product channel efficiencies for reaction 56. The structure of the $\mathrm{CHNO}$ isomer, formed as a co-product with $\mathrm{CO}$, may also have a significant bearing on the production of NO. It should be noted that recent experimental [51] and theoretical investigations $[52,53]$ demonstrate that $\mathrm{HCNO}+\mathrm{CO}$ and $\mathrm{HCN}+\mathrm{CO}_{2}$ are the major products, not $\mathrm{HOCN}+\mathrm{CO}$.

\section{CONCLUSIONS}

A mechanism describing the chemistry of nitrogenous species in combustion systems is reported, based where possible, on evaluated rate data. The simulations compare favorably with experimental measurements over a wide range of conditions. Detailed comparisons have also been made with simulations based on GRImech 3.0. Sensitivity analysis allows reactions important under different experimental conditions to be identified and compared with three other published reaction mechanisms, leading to the following conclusions:

- Significant differences exist between the rate coefficients and product channels of elementary reactions of nitrogenous species in four comprehensive reaction mechanisms for the prediction of NOx emissions from combustion systems. 
- The reactions showing high sensitivities and significant variations between mechanisms have been highlighted, thereby indicating where further experimental work is required to determine rate coefficients and product channels.

- In many cases, the number of experimental measurements for an individual reaction is small or covers only a limited temperature range. Often there are no evaluated rate coefficients and sometimes theoretical calculations of rate parameters show poor agreement with the experimental measurements.

- There are fewer fundamental studies for nitrogenous compounds in combustion systems than is the case for $\mathrm{C} / \mathrm{H} / \mathrm{O}$ species. This is especially true for intermediate species such as $\mathrm{CN}, \mathrm{NH}, \mathrm{NCO}, \mathrm{NNH}$, etc., where in general only profiles of relative concentrations are available. Further experimental studies of this type would greatly facilitate the evaluation of complex mechanisms and would provide a more demanding set of target data for optimization.

T. Turányi wishes to acknowledge the support of OTKA via contract T025875. A. S. Tomlin wishes to acknowledge the support of EPSRC.

\section{REFERENCES}

1. Glarborg, P., Alzueta, M. U., Dam-Johansen, K., and Miller, J. A., Combust. Flame 115:1 (1998).

2. Smith, G. P., Golden, D. M., Frenklach, M., Moriarty, N. W., Eiteneer, B., Goldenberg, M. C., Bowman, T., Hanson, R., Song, S., Gardiner, W. C. Jr., Lissianski, V., and Qin, Z., http://www.me.berkeley.edu/grimech.

3. Dean, A. M., and Bozzelli, J. W., in Gas-Phase Combustion Chemistry, $2^{\text {nd }}$. Edition, (W. C. Gardiner, Jr., Ed.), Springer-Verlag. in press.

4. Baulch, D. L., Cobos, C. J., Cox, R. A., Frank, P., Hayman, G., Just, Th., Kerr, J. A., Murrels, T., Pilling, M. J., Troe, J., Walker, R. W., and Warnatz, J., Combust. Flame 98:59 (1994).

5. Frenklach, M., Combust. Flame 58:69 (1984).

6. Frenklach, M., Wang, H., and Rabinowitz, M. J., Prog. Energy Combust. Sci. 18:47 (1992).

7. Frenklach, M., Wang, H., Bowman, C. T., Hanson, R. K., Smith, G. P., Golden, D. M., Gardiner, W. C., and Lissianski, V., Twenty-fifth Symposium (International) on Combustion, The Combustion Institute, Pittsburgh, Poster WIP-3-26, 1992.

8. Bowman, C. T., Hanson, R. K., Davidson, D. F., Gardiner, W. C., Jr., Lissianski, V., Frenklach, M., Goldenberg, M., Smith, G. P., Golden, D. M., and
Serauskas, R. V., Twenty-sixth Symposium (International) on Combustion, The Combustion Institute, Pittsburgh, Poster WIP-4-47, 1994.

9. Hughes, K. J., Turányi, T., Clague, A. R., and Pilling, M. J., Int. J. Chem. Kinet. submitted.

10. Pilling, M. J., Turányi, T., Hughes, K. J., and Tomlin, A. S., http://www.chem.leeds.ac.uk/Combustion/ Combustion.html\#MethMech.

11. Pilling, M. J., Turányi, T., Hughes, K. J., and Tomlin, A. S., http://www.chem.leeds.ac.uk/Combustion/ Combustion.html\#Nitrogen.

12. Miller, J. A., Branch, M. C., McLean, W. J., Chandler, D. W., Smooke, M. D., and Kee, R. J., Twentieth Symposium (International) on Combustion, The Combustion Institute, Pittsburgh, p. 673, 1984

13. Heard, D. E., Jeffries, J. B., Smith, G. P., and Crosley, D. R., Combust. Flame 88:137 (1992).

14. Williams, B. A., and Fleming, J. W., Combust. Flame 98:93 (1994).

15. Sausa, R. C., Anderson, W. R., Dayton, D. C., Faust, C. M., and Howard, S. L., Combust. Flame 94:407 (1993).

16. Harrington, J. E., Smith, G. P., Berg, P. A., Noble, A. R., Jeffries, J. B., and Crosley, D. R., Twenty-sixth Symposium (International) on Combustion, The Combustion Institute, Pittsburgh, p.2133, 1996

17. Glarborg, P., and Miller, J. A., Combust. Flame 99:475 (1994).

18. Bartok, W., Engelman, V. S., Goldstein, R., and del Valle, E. G., A.I.Ch.E. 126:30 (1972).

19. Steele, R. C., Malte, P. C., Nicol, D. G., and Kramlich, J. C., Combust. Flame 100:440 (1995).

20. Kee, R. J., Rupley, F. M., and Miller, J. A., ChemkinII. A Fortran Chemical Kinetics Package for the Analysis of Gas Phase Chemical Kinetics, Sandia National Laboratories Report No. SAND89-8009B, 1991.

21. Lutz, A. E., Kee, R. J., and Miller, J. A., Senkin. A Fortran Program for Predicting Homogeneous Gas Phase Chemical Kinetics with Sensitivity Analysis, Sandia National Laboratories Report No. SAND87-8248, 1988.

22. Glarborg, P., Kee, R. J., Grcar, J. F., and Miller, J. A., PSR. A Fortran Program for Modeling Well-Stirred Reactors, Sandia National Laboratories Report No. SAND86-8209, 1986.

23. Kee, R. J., Grcar, J. F., Smooke, M. D., and Miller, J. A., A Fortran Program for Modeling Steady Laminar One-Dimensional Premixed Flames, Sandia National Laboratories Report No. SAND85-8240, 1985.

24. Turányi, T., http://www.chem.leeds.ac.uk/Combustion/ Combustion.html.

25. Miller, J. A., and Melius, C. F., Twenty-fourth Symposium (International) on Combustion, The Combustion Institute, Pittsburgh,.., p. 719, 1992.

26. Mertens, J. D., Chang, A. Y., Hanson, R. K., and Bowman, C. T., Int. J. Chem. Kinet. 23:173 (1991).

27. Higashihara, T., Kuroda, H., Saito, K., and Murakami, I., Combust. Flame 61:167 (1985).

28: Hanson, R. K., and Salimian, S., in Combustion Chemistry (W. C. Gardiner Jr., Ed.), Springer-Verlag, New York, p.361, 1984. 
29. Mertens, J. D., Chang, A. Y., Hanson, R. K., and Bowman, C. T., Int. J. Chem. Kinet. 24:279 (1992).

30. Miller, J. A, and Bowman, C. T., Prog. Energy Combust. Sci. 15:287 (1989).

31. Marshall, P., Fontijn, A., and Melius, C. F., J. Chem. Phys. 86:5540 (1987).

32. Fujii, N., Sagawai, S., Sato, T., Nosaka, Y., and Miyama, H., J. Phys. Chem. 93:5474 (1989).

33. Bozzelli, J. W., and Dean, A. M., J. Phys. Chem. 93:1058 (1989).

34. Bozzelli, J. W., and Dean, A. M., Int. J. Chem. Kinet 27:1097 (1995).

35. Atakan, B., Wolfrum, J., and Weller, R., Ber. Bunsenges. Phys. Chem. 94:1372 (1990).

36. Bulatov, V. P., Ioffe, A. A., Lozovsky, V. A., and Sarkinov, O. M., Chem. Phys. Lett. 161:141 (1989).

37. Zuev, A. P., and Starikovskii, A. Yu., Khim. Fiz. 10:347 (1991).

38. Miller, J. A., and Bowman, C. T., Int. J. Chem. Kinet. 23:289 (1991)

39. Marshall, P., Ko, T., and Fontijn, A., J. Phys. Chem 93:1922 (1989).

40. Mebel, A. M., Lin, M. C., Morokuma, K., and Melius, C. F., Int. J. Chem. Kinet. 28:693 (1996).

41. Allen, M. T., Yetter, R. A., and Dryer, F. L., Int. J. Chem. Kinet. 27:883 (1995).

42. Jacobs, A., Wahl, M., Weller, R., and Wolfrum, J., Twenty-second Symposium (International) on Combus- tion, The Combustion Institute, Pittsburgh, p. 1093 , 1989

43. Woolridge, S. T., Hanson, R. K., and Bowman, C. T., Int. J. Chem. Kinet. 27:1075 (1995).

44. Cohen, N., and Westberg, K. R., J. Phys. Chem. Ref. Data 20:1211 (1991).

45. Glarborg, P., Dam-Johansen, K., and Miller, J. A., Int. J. Chem. Kinet. 27:1207 (1995).

46. Koizumi, H., Schatz, G. C., and Walch, S. P., J. Phys. Chem. 95:4130 (1991).

47. Miller, J. A., and Glarborg, P., Springer Ser. Chem. Phys. 61:318 (1996).

48. Temps, F., Wagner, H. Gg., and Wolf, M., Z. Phys. Chem. 176:27 (1992).

49. Unfried, K. G., Glass, G. P., and Curl, R. F., Chem. Phys. Lett. 177:33 (1991).

50. Boullart, W., Nguyen, M. T., and Peeters, J., J. Phys. Chem. 98:8036 (1994).

51. Eickhoff, U., and Temps, F., Phys. Chem. Chem. Phys. 1:243 (1999).

52. Miller, J. A., Durant, J. L., and Glarborg, P., Twentyseventh Symposium (International) on Combustion, The Combustion Institute, Pittsburgh, p. 235, 1998

53. Nguyen, M. T., Boullart, W., and Peeters, J., J. Phys. Chem. 98:8030 (1994).

Received 7 January 1999; revised 18 September 2000; accepted 30 September 2000 